\section{Ernährungssupplementation bei Magenkarzinom}

\author{
Gewichtsverlust und Mangelernährung belasten Patienten mit \\ fortgeschrittenem Magenkarzinom. Prävalenz, prognostische Bedeutung \\ und der Effekt einer Ernährungsunterstützung wurden nun untersucht.
}

D atienten mit einem Magenkarzinom sind besonders anfällig für Appetitverlust, Übelkeit und Erbrechen. Dies kann auch die Chemotherapie beeinträchtigen, denn gerade bei diesen Patienten entscheidet der Ernährungsstatus über die Verträglichkeit einer Chemotherapie. In einer zweiphasigen, nicht randomisierten Studie wurden die $\mathrm{Zu}$ sammenhänge untersucht. In der ersten Studienphase trugen die Forscher Daten zum Ernährungsstatus von Magenkarzinompatienten entsprechend den Kriterien des European Nutritional Risk Screenings (NRS) bei Patienten mit einem Adenokarzinom des Magens zusammen - erhoben innerhalb von $2 \mathrm{Ta}$ gen nach stationärer Aufnahme. Eine Mangelernährung wurde dabei bei ei- nem NRS-Score von 3 und höher unterstellt. In der zweiten Untersuchungsphase erhielten Patienten mit einem NRSStatus $\geq 3$ eine Ernährungsunterstützung. Bei schwachem Appetit wurde den Patienten Megestrolacetat angeboten, bei Schluckstörungen erhielten sie eine parenterale Ernährung.

In der ersten Studienphase wurden 830 Patienten behandelt, von denen 50,7\% einen NRS-Status von $\geq 3$ aufwiesen. Diese Patienten wiesen häufiger eine Stadium-IV-Erkrankung, erhöhte Werte von C-reaktivem Protein und eine Hyponatriämie auf. Das mediane Überleben war signifikant länger bei Patienten mit einem NRS $<3$ (31,9 vs. 25,7 Monate; $\mathrm{p}<0,001)$. In multivariaten Analysen erwies sich der NRS-Status als unabhängi- ger prognostischer Faktor. Die zweite Studienphase umfasste 347 Patienten, die eine palliative Chemotherapie erhielten. Innerhalb der ersten 3 Monate ging der NRS-Satus bei $17 \%$ von ihnen auf $<3$ zurück, nach 6 Monaten waren es $26,0 \%$. Junge Patienten, männliche Patienten und Patienten mit einem guten Ansprechen auf die Chemotherapie hatten eine größere Chance für einen NRS-Shift unter 3 nach der Ernährungssupplementation. Das mediane Überleben betrug 14,3 vs. 9,6 Monate zugunsten von Patienten mit einer Verbesserung des Ernährungsstatus $(\mathrm{p}=0,001)$.

Fazit: Magenkarzinompatienten im Stadium IV mit schlechtem Ernährungsstatus entsprechend dem European NRS profitieren hinsichtlich der Überlebenszeit von einer Ernährungssupplementation.

Barbara Kreutzkamp

Qiu M et al. Nutrition support can bring survival benefit to high nutrition risk gastric cancer patients who received chemotherapy. Support Care Cancer. 2015;23(7):1933-9.

\title{
Lymphomtherapie: Wann drohen Langzeitschäden?
}

\section{Lymphompatienten haben nach autologer Stammzelltransplantation mittlerweile eine sehr gute Prognose, sodass heute mögliche Langzeitfolgen in den Fokus des Interesses rücken. In einer Fall-Kontroll-Studie wurde nach therapieassoziierten Risikofaktoren gesucht.}

$\mathrm{H}$ ochdosis-Chemotherapie plus autologe Stammzelltransplantation (ASCT) ist eine Standardoption für Patienten mit rezidivierenden oder refraktären Lymphomen bzw. bisher unbehandelten Lymphomen mit hohem Rezidivrisiko. Im Kontext eines guten Langzeitüberlebens unter solchen Regimes rücken Fragen nach Spättoxizitäten in den Vordergrund. So haben Patienten nach autologer Stammzelltransplantation ein bis zu 3-fach erhöhtes Risiko für kardiovaskuläre Komplikationen.

In einer norwegischen Studie wurden deshalb die Prävalenz einer linksventrikulären systolischen Dysfunktion (LVSD) bei erwachsenen ehemaligen Lymphompatienten nach HochdosisChemotherapie plus ASCT sowie die da- für verantwortlichen Risikofaktoren im Vergleich zu alters- und geschlechtsmäßig passenden Kontrollen überprüft.

Einbezogen wurden 274 LymphomLangzeitüberlebende, die zwischen 1987 und 2008 behandelt worden waren, im Mittel mit einer kumulativen Doxorubicin-Dosis von $316 \mathrm{mg} / \mathrm{m}^{2}$. Bei $35 \%$ der Patienten war zusätzlich eine Strahlentherapie im Herzbereich durchgeführt worden. Nach einer durchschnittlichen Nachbeobachtungszeit von 13 Jahren diagnostizierten die Forscher bei 15,7\% der Patienten eine LVSD, 5,1\% waren asymptomatisch.

Die meisten Patienten mit symptomatischer Herzinsuffizienz hatten leichte Beschwerden, 8,8\% aller Patienten gruppierte man in die NYHA-Klasse II ein, höhere NYHA-Klassen wurden bei 1,8\% diagnostiziert. Verglichen mit den Kontrollen war das LVSD-Risiko der Ex-Lymphompatienten deutlich erhöht (Odds Ratio 6,6; $\mathrm{p}<0,001)$. Eine DoxorubicinDosis über $300 \mathrm{mg} / \mathrm{m}^{2}$ und eine Strahlentherapie im Herzbereich mit einer kumulativen Dosis von über 30 Gy erwiesen sich als unabhängige Risikofaktoren für eine LVSD.

Fazit: Langzeitüberlebende nach Hochdosis-Chemotherapie plus ASCT haben ein erhöhtes Risiko für eine linksventrikuläre Dysfunktion. Hohe Doxorubicinund Strahlen-Dosen erwiesen sich als unabhängige Risikofaktoren. Eine Modifikation dieser Faktoren könnte vielleicht helfen, die Rate der Langzeitschäden zu reduzieren. Barbara Kreutzkamp

Murbraech K et al. Heart failure and asymptomatic left ventricular systolic dysfunction in lymphoma survivors treated with autologous stem-cell transplantation: a national cross-sectional study. J Clin Oncol. 2015;33(24):2683-91. 